\title{
The Role of Blue Dye in Sentinel Node Detection for Breast Cancer: A Retrospective Study of 203 Patients
}

\author{
Jean-Remi Garbay Dounia Skalli-Chrisostome Nicolas Leymarie Benjamin Sarfati \\ Francoise Rimareix Chafika Mazouni \\ Institut Gustave Roussy, Villejuif, France
}

\section{Keywords}

Breast cancer - Sentinel lymph node - Surgery - Mapping . Blue dye $\cdot$ Radiolabeled colloid

\section{Summary}

Objective: We aimed at examining the potential benefits of blue dye in sentinel node biopsy (SNB) in comparison with its proven drawbacks. Patients and Methods: In 2007, 203 T1 primary breast carcinomas had been operated on in our institute. The patients had undergone a lumpectomy and SNB. Sentinel node (SN) detection was exclusively isotopic (ISO) in 77 patients and performed with blue dye combined with a radioactive isotope (COMBI) in 126 patients. We compared the number of SNs and the rate of SN positivity in both groups. Results: The detection rate was $99 \%$ in both groups: $76 / 77$ in the ISO group and 125/126 in the COMBI group. The mean number of $\mathrm{SNs}$ was 2.14 and 1.91 in the ISO group and the COMBI group, respectively (difference not significant (NS)). SN positivity was found in $26.1 \%$ and $24.6 \%$ in the ISO group and the COMBI group, respectively (NS). Only $1 \mathrm{SN}$ had been removed in $26 \%$ of the patients in the ISO group versus $45.2 \%$ of the patients in the COMBI group ( $p=0.004$ ). No significant differences were observed in the tumor characteristics. Conclusion: The systematic use of patent blue dye combined with isotopic detection does not appear to increase the overall performance of the SNB technique in this retrospective study.

(c) 2016 S. Karger GmbH, Freiburg

\section{Introduction}

The sentinel node biopsy (SNB) technique is now a standard technique for axillary nodal staging in primary N0 breast carcinomas measuring less than $30 \mathrm{~mm}[1,2]$. This technique allows better axillary staging than the classical lymphadenectomy and obviously fewer sequelae $[1,3,4]$.

The international community of surgeons conducted a rigorous evaluation of this technique, and methodological standards were established [5]. However, many technical details were not validated by randomized studies but are based on the opinions of experts from renowned reference centers. Thus, many variations still exist for sentinel node (SN) detection: For example, the number of injection sites varies between 1 and 4, and the site can be periareolar or not.

Right from the outset, experts recommended the combined technique with blue dye and an isotope for lymphatic mapping. The reasons will be discussed later in the article. However, blue dye has some major disadvantages:

- Allergic reactions occur in $0.5-2.7 \%$ of cases, with a mean value of $0.71 \%$ [6-8], often with severe reactions and some very rare deaths.

- The blue dye can stain the injection site for months and sometimes indefinitely.

- A deeply located blue lymph vessel may be difficult to find, and its dissection requires substantial expertise.

In order to avoid these disadvantages, some well-known teams switched to isotope detection alone, but provided limited new data. A controversy then arose about the systematic use of both tracers.

In this retrospective study, we compared the feasibility and performance of isotope detection alone (ISO) versus the classical combined method (COMBI). With the ISO method, the isotopic marker is used alone and detection is verified by the surgeon with a probe in the operating room. Blue dye is injected only in the event of percutaneous isotope detection failure. COMBI is the standard method using both tracers. 


\section{Patients and Methods}

We retrospectively analyzed 203 patients treated at the Gustave Roussy Cancer Campus between January 2007 and September 2007, for unifocal primary breast carcinoma (T1 N0) who had undergone a lumpectomy and SNB. Axillary clearance (AC) had been performed if the immediate histological analysis of the SN was positive or if the tumor size had attained $20 \mathrm{~mm}$, according to our protocol at that time. All procedures had been performed by senior surgeons or senior assistants. The ISO technique had been used in 77 patients and COMBI in 126 patients. The choice between the 2 methods was mainly linked to the surgeon, as some surgeons prefer one technique over the other. There was no other selection criterion.

Four subcutaneous injections of $0.2 \mathrm{ml}$ of rhenium colloidal sulfur (Nanocist $^{\star}$, CIS Bio International, Saclay, France) had been administered around the areola on the day before or on the day of the operation.

Most often, lymphoscintigraphy had not been performed, according to our protocol, to facilitate 1-day surgery. A CdTe probe (Gammed $2^{\mathrm{TM}}$, Eurorad, Strasbourg, France) had been used for perioperative detection.

Firstly, detection had been carried out by the surgeon through intact skin, in the operating room just after the induction of anesthesia.

In the ISO group, if isotope detection had failed, $2 \mathrm{ml}$ of patent blue dye (Lab. Gerbet, Paris, France) had been injected into 1 site subcutaneously. In the COMBI group, blue dye had been injected systematically. In both cases, the injection site was gently massaged over a few minutes. The axillary incision was made at least $10 \mathrm{~min}$ after the blue dye injection. The SN was defined as 'hot' when its isotopic activity was at least 3 -fold the background noise. The $\mathrm{SN}$ was defined as 'blue' when at least part of its surface was blue or when it was connected to a blue lymphatic channel.

All hot or blue nodes had been removed and up to 4 of them had been sent for an immediate pathological examination. The probe and manual palpation were used systematically to verify the nodes. Any suspicious palpable nodes were removed, identified as 'non-sentinel' and also sent for immediate examination.

If the tumor size had attained $20 \mathrm{~mm}$, AC had been systematically performed according to our protocol at that time. The SN was examined immediately by slide apposition and, eventually, by some limited frozen sections. Only the first 3 nodes were examined immediately.

The definitive pathological reports had been written following the American Joint Committee on Cancer (AJCC) tumor/node/metastasis (TNM) classification. When macrometastasis was found in the $\mathrm{SN}, \mathrm{AC}$ was performed systematically. If a micrometastasis or an isolated cell, i.e. pN1mi or pN0 i+, was found, inclusion in the randomized International Breast Cancer Study Group (IBCSG) trial B 23-01 comparing AC and follow-up was proposed to the patients. If the patient refused, $\mathrm{AC}$ was performed.

We compared the detection rate (DR) and histological results in both groups. The small population size and the short follow-up prohibited evaluation of the axillary recurrence rate and survival.

\section{Results}

The inclusion criteria for the SNB technique are described in table 1 . In both groups the mean age was $58.8 \pm 24$ years, with a homogeneous distribution of the cancer at various ages (table 2).

The body mass index (BMI) was comparable between the 2 groups: mean 25.7 (range 16.3-46.7). The tumor characteristics are described in detail in table 3 ; there was no significant difference between the 2 groups.

\section{Detection}

The SN had been identified successfully in $76 / 77$ cases in the ISO group and in $125 / 126$ cases in the COMBI group. Thus, the DR was $99 \%$ in both groups. The 2 patients with a detection failure
Table 1. Inclusion and exclusion criteria

\begin{tabular}{ll}
\hline Inclusion criteria & Exclusion criteria \\
\hline Invasive breast cancer & $\mathrm{N} 1$ or positive cytology \\
T1, unifocal tumor & $\mathrm{T} 2, \mathrm{~T} 3, \mathrm{~T} 4$ \\
N0 or negative cytology in case & multifocality \\
$\quad$ of a detectable axillary node & \\
Absence of neoadjuvant treatment & pregnancy or breast-feeding \\
Absence of previous breast or & allergic history \\
$\quad$ axillary surgery & patient's refusal \\
\hline
\end{tabular}

Table 2. Patient characteristics

\begin{tabular}{lll}
\hline & ISO & COMBI \\
\hline Mean age, years (range) & $58.8(34-81)$ & $58.8(32-86)$ \\
Patients, n (\%) & $77(100)$ & $126(100)$ \\
$\leq 45$ years & $9(11)$ & $19(15)$ \\
$46-55$ years & $24(31)$ & $30(24)$ \\
$56-65$ years & $22(29)$ & $42(33)$ \\
$>$ 65 years & $22(29)$ & $35(28)$ \\
BMI, kg/m2 (range) & $25.7(19.1-46.7)$ & $25.7(16.3-41.7)$ \\
Obese patients (BMI > 30), & $8(10)$ & $21(17)$ \\
n (\%) & & \\
\hline
\end{tabular}

ISO = Only isotope detection, COMBI = combined isotope and blue dye detection, $\mathrm{BMI}=$ body mass index.

Table 3. Breast tumor characteristics

\begin{tabular}{lcc}
\hline & ISO & COMBI \\
\hline Mean tumor size, mm & $11(5-20)$ & $11(4-20)$ \\
$\leq 10 \mathrm{~mm}$ & $29(38 \%)$ & $41(33 \%)$ \\
$11-15 \mathrm{~mm}$ & $17(22 \%)$ & $28(22 \%)$ \\
$16-20 \mathrm{~mm}$ & $6(8 \%)$ & $15(12 \%)$ \\
Location & & \\
External quadrants & $48(62 \%)$ & $60(48 \%)$ \\
Internal quadrants & $17(22 \%)$ & $41(32 \%)$ \\
Central quadrants & $12(16 \%)$ & $25(20 \%)$ \\
Histology & & $80(63 \%)$ \\
Infiltrating ductal carcinoma & $56(73 \%)$ & $10(8 \%)$ \\
Infiltrating lobular carcinoma & $6(8 \%)$ & $31(25 \%)$ \\
DCIS microinfiltrating & $14(18 \%)$ & $5(4 \%)$ \\
Other types & $1(1 \%)$ & $36(39 \%)$ \\
EE histologic grade & & $41(44 \%)$ \\
Grade I & $26(42 \%)$ & $16(17 \%)$ \\
Grade II & $24(39 \%)$ & $12(19 \%)$ \\
Grade III & $12 \%$ & \\
\hline
\end{tabular}

ISO = Only isotope detection, $\mathrm{COMBI}=$ combined isotope and blue dye detection, DCIS = ductal carcinoma in situ, EE = Elston and Ellis.

had undergone AC. In the COMBI group, the patient was 61 years old, with a BMI of 26 . The tumor was a 10 -mm grade II infiltrating ductal carcinoma. There were $8 / 20$ positive nodes at AC. In the ISO group, the patient was 51 years old with a BMI of 34 . The tumor was a $14-\mathrm{mm}$ grade III infiltrating ductal carcinoma. There 
Table 4. Sentinel node detection

\begin{tabular}{cccc}
\hline & ISO & COMBI & p \\
\hline Detection rate, n (\%) & $76(99)$ & $125(99)$ & NS \\
Mean number of SNs (range) & $2.1(1-5)$ & $1.9(1-7)$ & NS \\
Sentinel nodes, n (\%) & 76 & 125 & \\
1 & $20(26)$ & $57(46)$ & 0.004 \\
2 & $33(43)$ & $34(27)$ & \\
$\geq 3$ & $23(31)$ & $34(27)$ & \\
\hline
\end{tabular}

$\mathrm{ISO}=$ Only isotope detection, $\mathrm{COMBI}=$ combined isotope and blue dye detection, $\mathrm{SN}=$ sentinel node, $\mathrm{NS}=$ not significant.

Table 5. Sentinel node status

\begin{tabular}{llll}
\hline & ISO & COMBI & $\mathrm{p}$ \\
\hline Patients, n (\%) & & & \\
$\quad$ Negative SN & $57(74)$ & $94(75)$ & $\mathrm{NS}$ \\
$\quad$ Micrometastasis & $10(13)$ & $17(13)$ & $\mathrm{NS}$ \\
$\quad$ Macrometastasis & $10(13)$ & $15(12)$ & $\mathrm{NS}$ \\
Involved nodes (SN+), n (\%) & $43 / 165(26)$ & $58 / 241(24)$ & $\mathrm{NS}$ \\
$\quad$ Preoperative SN+ & $12 / 165(7)$ & $20 / 241(8)$ & $\mathrm{NS}$ \\
$\quad$ Definitive SN+ & $31 / 165(19)$ & $38 / 241(16)$ & $\mathrm{NS}$ \\
\hline
\end{tabular}

ISO = Only isotope detection, $\mathrm{COMBI}=$ combined isotope and blue dye detection, $\mathrm{SN}=$ sentinel node, $\mathrm{NS}=$ not significant.

were no positive nodes among the 7 nodes examined at AC. In the ISO group, isotope detection had been unsuccessful in 11 patients (12.5\%) and injection of the patent blue dye had been performed. During surgery, 10/11 patients had blue nodes and 5 of them were also hot. In this group of patients in whom isotope detection had failed, there were more obese women (BMI > 30): $36.4 \%$ versus $14 \%(\mathrm{p}=0.06)$, but not a greater number of elderly patients $(>60$ years): $64 \%$ versus $60 \%$ (not significantly different (NS)).

\section{Number of SNs}

The mean number of SNs was identical in the 2 groups: 2.1 (1-5) for ISO and 1.9 (1-7) for COMBI. However, there were significantly more patients with only $1 \mathrm{SN}$ removed in the COMBI group compared to the ISO group: $46 \%$ versus $26 \%$, respectively (p $=0.004)$ (table 4).

\section{Number of Non-Sentinel Nodes}

Any suspicious palpable node was identified as 'non-sentinel' and removed: $6 / 77$ patients (8\%) for the ISO group and 12/126 (10\%) for the COMBI group.

\section{Histology}

Immediate analysis of the SNs had unveiled metastases in 12/65 nodes $(7.3 \%)$ and in 20/241 nodes (8.3\%) in the ISO and COMBI group, respectively (NS). They were all macrometastases.

In the final analysis, the SN was positive in $26 \%$ and $25 \%$ of the patients in the ISO and COMBI group, respectively (NS). Micrometastasis had been found in $13 \%$ of the patients in both groups (table 5).
Table 6. Nodal status related to age and tumor size

\begin{tabular}{llrl}
\hline & ISO & COMBI & $\mathrm{p}$ \\
\hline $\begin{array}{l}\text { Positive SN, n (\%) } \\
\leq 45 \text { years }\end{array}$ & $2(22)$ & $5(26)$ & NS \\
46-55 years & $8(33)$ & $7(22)$ & NS \\
$56-65$ years & $3(13)$ & $10(24)$ & NS \\
$>65$ years & $7(32)$ & $10(28)$ & NS \\
Positive SN, n (\%) & & & \\
T $\leq 10 \mathrm{~mm}$ & $9(27)$ & $11(22)$ & NS \\
$11-15 \mathrm{~mm}$ & $7(35)$ & $13(39)$ & NS \\
$16-20 \mathrm{~mm}$ & $4(50)$ & $8(44)$ & NS \\
\hline
\end{tabular}

ISO = Only isotope detection, COMBI = combined isotope and blue dye detection, $\mathrm{SN}$ = sentinel node, $\mathrm{NS}=$ not significant.

The levels of SN involvement according to clinical parameters (age, tumor size) are described in table 6. There was no significant difference.

When the SN was negative, none of the non-sentinel nodes was positive.

\section{Discussion}

The large studies conducted to validate the SN technique always used both tracers $[1,4]$. But the performance of isotope detection alone is excellent. Gradually, because of the risks observed with blue dye, the use of isotope alone gained momentum. This retrospective study, with a literature review, aims at reviewing this debatable option.

\section{Detection Rate}

In our series, the DR was $99 \%$ in both groups, with only 1 failure in each group. These 2 failures were related to an extensive nodal involvement in the COMBI group and to obesity (BMI of 34) in the ISO group.

Some series indicate that each tracer alone yields a lower DR than the combined technique [4]. In the meta-analysis published by Kim et al. in 2005 [9], the mean DR was $83.1 \%$ with blue dye alone and $96.4 \%$ with both tracers. However, in the most recent series, the DR with the isotope alone or combined with blue dye was always excellent and very close, between $93.5 \%$ and $97.2 \%$ [4, 9-11]. The sole randomized trial comparing ISO and COMBI was published recently, with 667 patients [12]. This study failed to demonstrate any advantage with COMBI in the presence of a positive lymphoscintigram. So, given the published results, we can safely assume that there is no DR benefit with COMBI.

\section{False-Negative Rate and Oncologic Safety}

Until 2010, the false-negative (FN) rate was considered the main evaluation criterion for the routine use of the SNB technique. A meta-analysis of 69 trials showed a mean $\mathrm{FN}$ rate of $8.4 \%$ in 8,059 patients (5.5-16.7\%), most often with combined tracers [9]. This rate was sufficiently satisfactory to allow the routine use of the 
SNB technique, but clinicians remain dubious about the true consequences of this $8.4 \% \mathrm{FN}$ rate. At that point in time, we could not exclude the risk of an increase in the FN rate with isotope detection alone. That is why all recommendations up to 2010 underlined the use of both tracers and were reluctant to dispense with the blue dye.

However, in many publications, the topographic concordance between the blue dye and the isotope was excellent [13], indicating that most often both tracers reach the same SN, which was found to be blue and hot.

In a large series, only $0.8 \%(4 / 496)$ of the patients who were SN positive had a blue-only node as their only positive node [2].

In 2010, the National Surgical Adjuvant Breast and Bowel Project (NSABP) B32 trial comparing SNB to AC in 5,611 patients [4] indicated identical overall survival at 8 years: $90.3 \%$ and $91.8 \%$, respectively (NS). The axillary recurrence rate was very low and also identical in the 2 arms: $0.7 \%$ and $0.4 \%$, respectively (NS). This very low axillary recurrence rate is far lower than would be expected with an FN rate of $7-10 \%$. This study therefore demonstrates that the clinical significance of the FN rate is really very low, with only $0.7 \%$ axillary recurrence at 8 years for 2,800 patients in the SNB arm. Thus, even if the ISO option could increase the FN rate (which is not proven), would this have any impact on the axillary recurrence rate? This impact is obviously nil or very low, but in the absence of a randomized comparison with systematic lymphadenectomy, it is not possible to come to a definitive conclusion.

\section{Number of SNs Removed}

The number of SNs removed is an important quality indicator of the technique $[4,10]$. The FN rate decreases with the number of $\mathrm{SN}$ removed for up to 4 nodes [9]. The usual recommendation is to remove all blue and/or hot nodes, up to 4 [14].

However, the ideal mean number should be around 2 [15]. In our study, the mean number of nodes removed was similar and in accordance with the recommended standards, with 2.14 in the ISO group and 1.91 in the COMBI group (NS). Significantly more patients had only $1 \mathrm{SN}$ removed in the COMBI group (46\%) than in the ISO group $(26 \%)(\mathrm{p}=0.004)$ (table 4$)$. These results indicate that the ISO technique is not associated with a significant reduction in the number of SNs removed. The same results were found in the randomized trial [12].

\section{The Non-Sentinel Nodes}

Only highly suspicious palpable nodes were removed during the SNB procedure. The number of non-sentinel nodes was low and equivalent in both arms ( $8 \%$ and $10 \%)$. When the SN was negative, none of the non-sentinel nodes was positive. One could suppose that the surgeon would rather remove more nodes in the ISO group in order to improve the felt low DRs, but this was not the case.

\section{Histological Nodal Status}

The rate of SN invasion was similar in the 2 groups, ISO and COMBI, with $26.1 \%$ and $24.1 \%$, respectively. This is in accordance with other larger series [4] and with the randomized trial [12].

\section{Drawbacks of Blue Dye}

The main drawback is the risk of allergic reactions, from a simple skin rash to an anaphylactic shock $[6-8,16]$, with a risk of death. This risk is unpredictable, and not related to atopic history [7, 17].

The second drawback is a persistent subcutaneous blue stain. This is observed in $70 \%$ of the patients at 3 months, and in $41 \%$ at 1 year with a subdermal injection [18]. In this study, all blue dye injections were made deeper in the subcutaneous plane and all blue staining of the skin had disappeared at 3 months.

\section{Surgical Technique}

There is a very significant technical point concerning SN dissection which is rarely discussed: At the beginning of surgery, each surgeon has to choose which tracer he wants to follow first, as the dissection technique is totally different. Later on, the surgeon will move frequently from one tracer to the other, but a choice has to be made at the beginning:

With the blue dye first, a large skin incision is required and a very accurate dissection is needed to find the blue channel without cutting it and then track the progression of the tracer up to the blue node. This can be more or less rapid. This procedure can lead to a wide dissection, which is associated with a higher risk of functional sequelae.

With isotope detection first, a real learning curve is needed for proper handling of the probe [19]. But thereafter, isotope detection would be easy and quick: The skin incision will be smaller, just in front of the detected signal, and the dissection will be minimal, following the probe. When the hot node is found, it is often blue with its blue channel, which is reassuring but finally of no use.

In our experience, dissection with isotope detection very rapidly became preponderant. When all hot SNs have been removed, the surgeon will be very reluctant to extend the surgical dissection in search of a hypothetical additional blue-only node. In the end, the blue dye injection has not helped improve this frequent situation.

\section{Learning Curve}

Conversely, during the learning curve, both tracers should be used systematically to improve the overall performance during this training period, and also to enable the surgeon to be at ease with the blue dye if isotope failure occurs.

\section{Conclusion}

In this study, systematic addition of the blue dye did not modify the overall performance of SNB, compared to isotopic detection alone in the presence of a positive percutaneous detection. This is in agreement with all published results $[2,12,15]$.

So, to dispense with the blue dye in the presence of a positive lymphoscintigram or percutaneous detection is, for us, a valid option.

\section{Disclosure Statement}

The authors declare no conflicts of interest and no sponsorship. 


\section{References}

1 Veronesi U, Paganelli G, Viale G, et al.: A randomized comparison of sentinel-node biopsy with routine axillary dissection in breast cancer. N Engl J Med 2003; 349:546-553.

2 Kang T, Yi M, Hunt KK, et al.: Does blue dye contribute to success of sentinel node mapping for breast cancer? Ann Surg Oncol 2010;(suppl 3):280-285.

$\checkmark 3$ Wilke LG, McCall LM, Posther KE, et al.: Surgical complications associated with sentinel lymph node biopsy: results from a prospective international cooperative group trial. Ann Surg Oncol 2006;13:491-500.

$\checkmark 4$ Krag DN, Anderson SJ, Julian TB, et al.: Sentinel lymph-node resection compared with conventional axillary-lymph-node dissection in clinically node-negative patients with breast cancer: overall survival findings from the NSABP B-32 randomised phase 3 trial. Lancet Oncol 2010;11:927-933.

5 Schwartz GF: Clinical practice guidelines for the use of axillary sentinel lymph node biopsy in carcinoma of the breast: current update. Breast J 2004;10:85-88.

6 Albo D, Wayne JD, Hunt KK, et al.: Anaphylactic reactions to isosulfan blue dye during sentinel lymph node biopsy for breast cancer. Am J Surg 2001;182:393-398.

7 Howard JD, Moo V, Sivalingamt P: Anaphylaxis and other adverse reactions to blue dyes: a case series. Anaesth Intensive Care 2011;39:287-292.
Bézu C, Coutant C, Salengro A, et al.: Anaphylactic response to blue dye during sentinel lymph node biopsy. Surg Oncol 2011;20:e55-e59.

9 Kim T, Giuliano AE, Lyman GH: Lymphatic mapping and sentinel lymph node biopsy in early-stage breast carcinoma. Cancer 2006;106:4-16.

10 Goyal A, Newcombe RG, Chhabra A, et al.: Factors affecting failed localisation and false-negative rates of sentinel node biopsy in breast cancer - results of the ALMANAC validation phase. Breast Cancer Res Treat 2006;99:203-208.

11 Gill G: Sentinel-lymph-node-based management or routine axillary clearance? One-year outcomes of sentinel node biopsy versus axillary clearance (SNAC): a randomized controlled surgical trial. Ann Surg Oncol 2009;16:266-275.

12 O’Reilly EA, Prichard RS, Al Azawi D, et al.: The value of isosulfan blue dye in addition to isotope scanning in the identification of the sentinel lymph node in breast cancer patients with a positive lymphoscintigraphy: a randomized controlled trial. Ann Surg 2015;262:243248.

13 Kern KA: Concordance and validation study of sentinel lymph node biopsy for breast cancer using subareolar injection of blue dye and technetium $99 \mathrm{~m}$ sulfur colloid. J Am Coll Surg 2002;194:467-475.
4 Gill J, Lovegrove R, Naessens K, et al.: Sentinel lymph node biopsy in breast cancer: an analysis of the maximum number of nodes requiring excision. Breast J 2001;17:3-8.

15 Schuman S, Walker G, Avisar E: Processing sentinel nodes in breast cancer. When and how many? Arch Surg 2011;146:389-393.

16 Bines S, Kopkash K, Ali A, et al.: The use of radioisotope combined with isosulfan blue dye is not superior to radioisotope alone for the identification of sentinel lymph nodes in patients with breast cancer. Surgery 2008;144:606-609.

17 Raut CP, Daley MD, Hunt KK, et al.: Anaphylactoid reactions to isosulfan blue dye during breast cancer lymphatic mapping in patients given preoperative prophylaxis. J Clin Oncol 2004;22:567-568.

18 Govaert GAM, Oostenbroek RJ, Plaisier PW: Prolonged skin staining after intradermal use of patent blue in sentinel lymph node biopsy for breast cancer. Eur J Surg Oncol 2005;31:373-375

19 Derossis AM, Fey J, Yeung H, et al.: A trend analysis of the relative value of blue dye and isotope localization in 2000 consecutive cases of sentinel node biopsy for breast cancer. J Am Coll Surg 2001;193:473-478. 